Chapman University

Chapman University Digital Commons

Physical Therapy Faculty Articles and Research

Physical Therapy

2014

\title{
Potential for Using Smartphone Accelerometers in Non-laboratory Environments
}

\author{
Rahul Soangra \\ ChapmanUniversity, soangra@chapman.edu
}

Thurmon Lockhart

Virginia Polytechnic Institute and State University

Christopher W. Frames

Virginia Tech

Jian Zhang

Virginia Tech

Seong Hyun Moon

Virginia Tech

See next page for additional authors

Follow this and additional works at: https://digitalcommons.chapman.edu/pt_articles

Part of the Movement and Mind-Body Therapies Commons, Musculoskeletal System Commons, and the Physical Therapy Commons

\section{Recommended Citation}

Soangra R, Lockhart TE, Frames CW, Zhang J, Moon SH, \& Park J. Potential for using smartphone accelerometers in non-laboratory environments. Proc Hum Factors Ergon Soc Annu Meet. 2014;58(1):1672-1675. doi: 10.1177/1541931214581349

This Conference Proceeding is brought to you for free and open access by the Physical Therapy at Chapman University Digital Commons. It has been accepted for inclusion in Physical Therapy Faculty Articles and Research by an authorized administrator of Chapman University Digital Commons. For more information, please contact laughtin@chapman.edu. 


\section{Potential for Using Smartphone Accelerometers in Non-laboratory Environments}

\section{Comments}

This article was originally published in Proceedings of the Human Factors and Ergonomics Society Annual

Meeting, volume 58, issue 1, in 2014. DOI: 10.1177/1541931214581349

\section{Copyright}

Not subject to U.S. copyright restrictions.

\section{Authors}

Rahul Soangra, Thurmon Lockhart, Christopher W. Frames, Jian Zhang, Seong Hyun Moon, and Jongsoon Park 


\title{
Potential for using Smartphone Accelerometers in Non- laboratory Environments
}

\author{
Rahul Soangra ${ }^{1,2}$, Thurmon E. Lockhart ${ }^{1,2}$, Christopher W. Frames ${ }^{1}$, Jian Zhang ${ }^{1}$, Seong Hyun Moon ${ }^{1}$, \\ Jongsoon Park ${ }^{1}$ \\ 1. Industrial \& Systems Engineering, Virginia Tech 2. School of Biomedical Engineering and \\ Sciences, Virginia Tech-Wake Forest University
}

\begin{abstract}
In recent years, there has been rising interest in using accelerometers as an alternative instrument to measure the center of pressure. Accelerometers are inexpensive, small, sensitive, and can be readily used in non-laboratory environments. In addition to this, smartphones with built-in accelerometers add to their capability with real-time processing of data from sensors. Clinicians and researchers are currently in disagreement from whether these measurements provide the same physiological information about the participant's balance. In this study, twelve participants were asked to wear smartphone on their right ASIS using a belt clip and stand still on the forceplate. The data was synchronized using a tap by the right foot and collected over a fifty second period for analysis. Various linear and non-linear measures were extracted from the time series of resultant principal component (PC) scores. The results show high correlation in the $\mathrm{COP}$ time series from the two instruments $(\mathrm{R}=0.86)$.
\end{abstract}

\section{Introduction}

Static balance is traditionally quantified using measures of sway during quiet standing. Stabilometry representing excursions of Center of Pressure (COP) is considered to be the most important measure in quantifying postural steadiness. Recently exploratory studies on COP from inertial sensors (accelerometers in particular) have been reported (Mayagoitia, Lotters, Veltink, \& Hermens, 2002). Accelerometers being smaller, less expensive, and more sensitive sensors have potential advantages over forceplates as they have greater applicability outside laboratories. Adlerton discovered that trunk accelerations correlated better with COP excursions from forceplates and increased in amplitudes during onelegged stance fatigue (Adlerton, Moritz, \& Moe-Nilssen, 2003). Some authors have reported accelerometers to be highly sensitive and reliable in differentiating Parkinson's disease patients and healthy controls (Mancini et al., 2011; Mancini et al., 2012; Rocchi, Chiari, Cappello, \& Horak, 2006) which has been validated by Mancini (Mancini et al., 2012). Despite of the attempts by several authors to establish a relationship between forceplate COP and accelerometers(Adlerton et al., 2003; Najafi et al., 2010; Whitney et al., 2011), the complexity of this task is caused from the fact that forceplates and accelerometers have their own local sensitive axes, which this makes the comparison between the two devices difficult as the mounting of accelerometers and human standing foot direction with respect to forceplate axes needs to be accurately controlled.

Nowadays, it is known that all the latest smartphones available in market are embedded with accelerometers. Smartphones have advantages as they can readily process the raw data and convert it into a clinically understandable form. Smartphone technology is quite promising and provides a versatile platform in practical usage of accelerometers for balance assessment, already reported to have high reliability, sensitivity and validity.(Mancini \& Horak, 2010; MoeNilssen, Nordin, Lundin-Olsson, \& Work Package 3 of
European Community Research Network Prevention of Falls Network, 2008), smartphone technology is quite promising and provides a versatile platform for research purposes.

Yet in the research world there is still no consensus as to which sway parameter is comparable between accelerometers and forceplates. Some researcher emphasized on root mean square (RMS) of acceleration signals (Amiridis, Hatzitaki, \& Arabatzi, 2003; Maki, Holliday, \& Fernie, 1990; Prieto, Myklebust, Hoffmann, Lovett, \& Myklebust, 1996) and Jerk(Mancini et al., 2011). The objective of this study is to establish a relationship between COP measures from forceplates and smartphone accelerometers. To achieve this goal, we measured forceplate and smartphone data concurrently and compared them for linear and non-linear physiological variable relationships. As it seemed practically impossible to align axes of a smartphone and that of forceplate, we conducted principal component analysis of the $\mathrm{COP}$ coordinates of the two systems.

\section{Methods \\ Subjects}

Twelve subjects (6 males, 6 females) with no previous history of neurological disorders participated in this study. The average age of participants were $26 \pm 4$ years (average \pm SD), height was $162 \pm 9 \mathrm{~cm}$, and weight was $68 \pm 17 \mathrm{~kg}$. The protocol involved three trials for each subject. All subjects had to provide written consent as per Virginia Tech Institutional Review Board (VT-IRB).

\section{Equipment}

The experimental set-up consisted of three 60 seconds trials for each subject. Subject stood on a forceplate, with eyesopen, looking forward towards a target $4 \mathrm{~m}$ away from the subject. 
In the first 10 seconds of data collection, the participants were asked to tap their right foot. Both the forceplate and accelerometer data were not filtered. From the output signals of the smartphone and forceplate, the signals were matched at the peaks and truncated 50 seconds after the tapping peak. The sampling frequency of the forceplate was kept as $100 \mathrm{~Hz}$ and smartphone at $30 \mathrm{~Hz}$. The forceplate data was resampled to $30 \mathrm{~Hz}$ for further analysis. From the truncated data (50 seconds), the two COP coordinates were computed in anteriorposterior (AP) and medio-lateral (ML) directions for forceplates (COPx and COPy) and accelerometers (Acc COPX and Acc COPy).

\section{Principal component Analysis (PCA)}

It seemed practically impossible to align local axes of smartphone and that of forceplate. PC were more meaningful than COP trajectories since they were aligned with the maximum variability of human sway (figure 1). The PCA procedure was applied to COP coordinates from both forceplates and accelerometers. The covariance matrix was used to estimate Principal Components (PCs). The two eigen vectors were used as new directions. These directions depended on maximum variability (higher eigen value) direction of the COP. As the sway data was collected concurrently from the two instruments it can be assumed that both smartphone and forceplates have a common direction of maximum sway variability. Thus in this study we calculated resultant PC score as defined below

$$
\text { Resultanc } P C \text { scorte }=\sqrt{\left((P C 1 \text { score })^{2}+(P C 2 \text { scort } \theta)^{2}\right)}
$$

The times series of resultant PC score from both instruments were used for further analysis.

\section{Results}

All the results were derived using time series of resultant principal component score from the two systems as derived in equation 1. The results show high correlation in the COP time series from the two instruments $(\mathrm{R}=0.86)$ (figure 2). Mean radius, area and total excursion path of the stabilogram using resultant PC scores was found to be higher for the smartphone than that of forceplate $(p<0.01)$. A higher frequency was found in the forceplate signals than in smartphone for the same physiological signals. Non-linear analysis of signals revealed that there was no significant difference in scaling exponent (alpha) found in signals between the forceplate and smartphone. However, the sample entropy (complexity) was higher in forceplate signals than in smartphone signals.
Table 1: Various parameters derived from forceplate and smartphone time series

\begin{tabular}{|c|c|c|c|c|c|c|}
\hline & \multicolumn{6}{|c|}{ Instuments } \\
\hline & \multicolumn{2}{|c|}{$\begin{array}{l}\text { Forceplate\& } \\
\text { Smatphone }\end{array}$} & \multicolumn{2}{|c|}{ Forceplats } & \multicolumn{2}{|c|}{ Smarphone } \\
\hline Paramaters & सеап & St Dev & मөबar & St DeV & Bhean & Stad DE \\
\hline Jpha & & & 0.88 & 0.25 & 0.92 & 020 \\
\hline 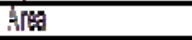 & & & 2434 & 27.42 & 412.57 & 36.54 \\
\hline $\begin{array}{l}\text { Tros8Comelation } \\
\text { Sosfifigient }\end{array}$ & 0.88 & 0.07 & & & & \\
\hline Hzxinum Frequenc; & & & 0.00 & 0.03 & 0.04 & 0.02 \\
\hline sathLenth & & & 52.73 & 5.45 & 1006.92 & 27.106 \\
\hline Jors & & & 5.97 & $8.4]$ & 8.81 & 7.89 \\
\hline zalitils & & & 7,33 & 3.81 & 10.77 & 398 \\
\hline Sampe Ertory & & & 3.34 & 0.35 & 3.82 & 0.26 \\
\hline
\end{tabular}
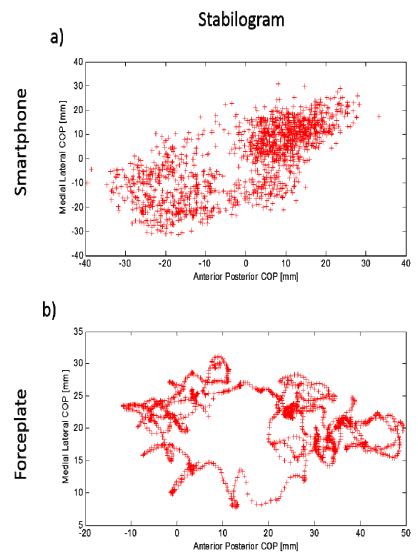

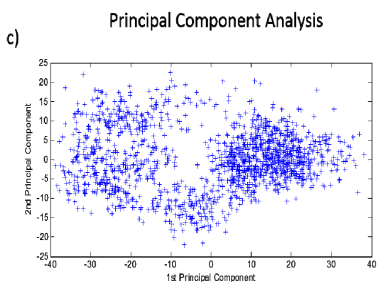

d)

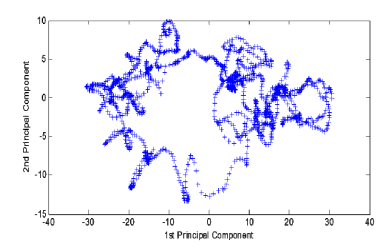

Figure 1: Sample data of a participant's stabilogram from (a) smartphone and (b) forceplate and principal components (c) smartphone and (d) forceplate system

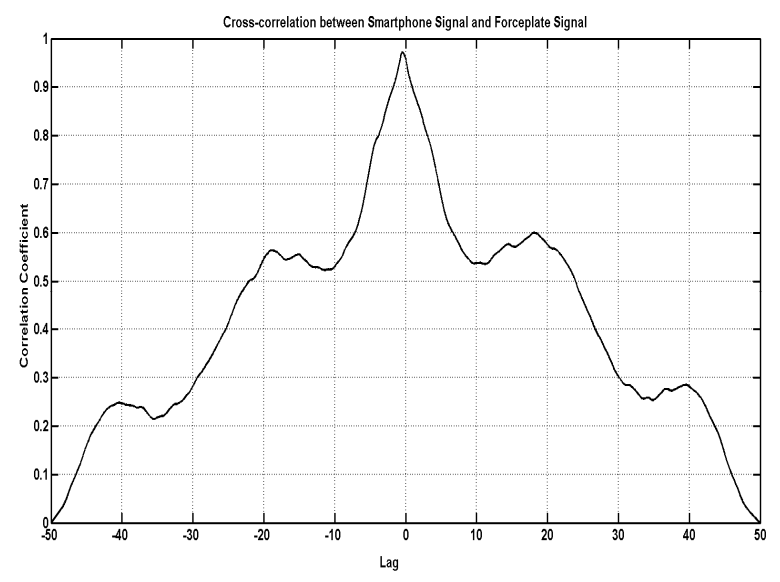

Figure 2: Sample data of cross-correlation between resultant PC signals from smartphone and forceplate 

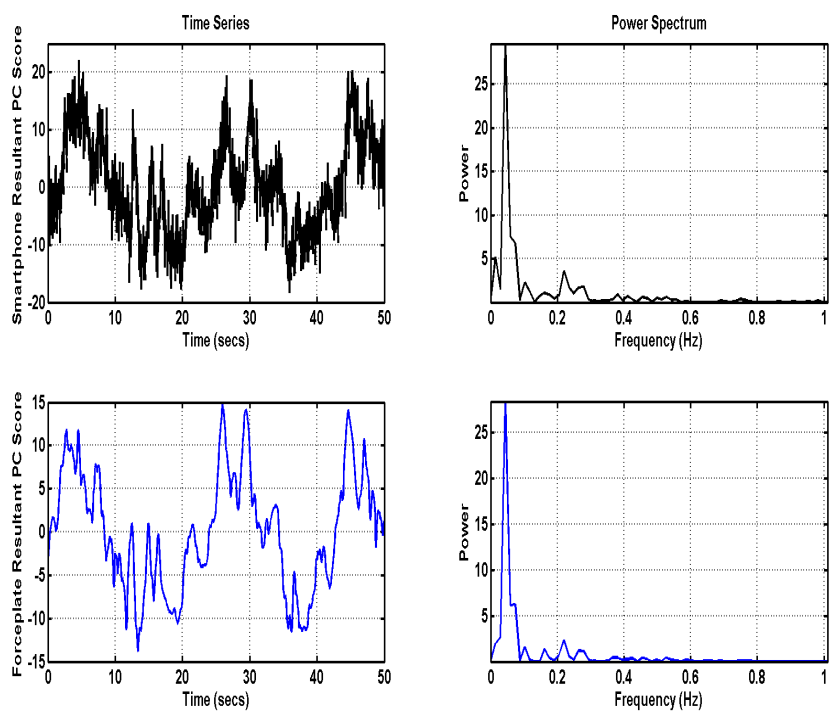

Figure 3: Sample data of the participant's resultant PC score signals and its power spectrum

\section{Discussion}

To our knowledge there has been no previous studies examining concurrent intra-individual relationship between COP derived from accelerometer and forceplate. COP measurement can be time consuming to administer using both forceplates and accelerometers, this may require specialist training. On the other hand smartphone could be a viable alternative in the measurement of COP. The preliminary results indicate the feasibility of using calculated parameter resultant PC score. The resultant PCs that were derived from the collected time series data from two systems were highly correlated $(\mathrm{R}=0.86)$ (figure 2$)$. Linear measures such as mean radius, area and total excursion path of stabilogram using resultant PC scores were found to be higher for smartphone than from the forceplate, which may be attributed to the high level of noise in smartphone signals (figure 3). It was also seen that higher frequencies were found in forceplate signals than in smartphone for the same physiological signals collected simultaneously. This can be explained to some extent by the position of smartphone, situated at waistline and our propensity to maintain body orientations for head stabilization (Assaiante \& Amblard, 1993; Bril \& Ledebt, 1998; Pozzo, Berthoz, \& Lefort, 1990). This may also be partially explained by the fact that the forceplate data was collected at $100 \mathrm{~Hz}$ and higher frequency signals could have been sampled versus the $30 \mathrm{~Hz}$ of the smartphone. Non-linear analysis revealed that complexity of signals from the forceplate was higher, which may be attributed to the presence of higher degrees of freedom from joints such as knee, ankle and hip. The traditional approaches for the analysis of center of pressure are focused on linear variables such as COP path length and area. These variables show changes as function of manipulations in environmental and organismic constraints but fail to reveal time evolutionary properties of center of pressure. The non-linear measures such as sample entropy and scaling exponent were determined for time series signals from both instruments. One of the challenges that arise is the alignment of axes in forceplate and smartphone for standing human postures. In order to align axes between the two instruments, we conducted principal component analysis of the COP coordinates of the two systems. Both systems showed higher correlation in resultant PC score time series signals for both linear and non-linear sway information.

\section{References}

Adlerton, A. K., Moritz, U., \& Moe-Nilssen, R. (2003). Forceplate and accelerometer measures for evaluating the effect of muscle fatigue on postural control during one-legged stance. Physiother Res Int, 8(4), 187-199.

Amiridis, I. G., Hatzitaki, V., \& Arabatzi, F. (2003). Ageinduced modifications of static postural control in humans. Neurosci Lett, 350(3), 137-140.

Assaiante, C., \& Amblard, B. (1993). Ontogenesis of head stabilization in space during locomotion in children: influence of visual cues. Exp Brain Res, 93(3), 499515.

Bril, B., \& Ledebt, A. (1998). Head coordination as a means to assist sensory integration in learning to walk. Neurosci Biobehav Rev, 22(4), 555-563.

Maki, B. E., Holliday, P. J., \& Fernie, G. R. (1990). Aging and postural control. A comparison of spontaneous- and induced-sway balance tests. J Am Geriatr Soc, 38(1), $1-9$.

Mancini, M., \& Horak, F. B. (2010). The relevance of clinical balance assessment tools to differentiate balance deficits. Eur J Phys Rehabil Med, 46(2), 239-248.

Mancini, M., Horak, F. B., Zampieri, C., Carlson-Kuhta, P., Nutt, J. G., \& Chiari, L. (2011). Trunk accelerometry reveals postural instability in untreated Parkinson's disease. Parkinsonism Relat Disord, 17(7), 557-562. doi: 10.1016/j.parkreldis.2011.05.010

Mancini, M., Salarian, A., Carlson-Kuhta, P., Zampieri, C., King, L., Chiari, L., \& Horak, F. B. (2012). ISway: a sensitive, valid and reliable measure of postural control. J Neuroeng Rehabil, 9, 59. doi: 10.1186/1743-0003-9-59

Mayagoitia, R. E., Lotters, J. C., Veltink, P. H., \& Hermens, H. (2002). Standing balance evaluation using a triaxial accelerometer. Gait Posture, 16(1), 55-59.

Moe-Nilssen, R., Nordin, E., Lundin-Olsson, L., \& Work Package 3 of European Community Research Network Prevention of Falls Network, E. (2008). Criteria for evaluation of measurement properties of clinical balance measures for use in fall prevention studies. J Eval Clin Pract, 14(2), 236-240. doi: 10.1111/j.1365-2753.2007.00839.x

Najafi, B., Horn, D., Marclay, S., Crews, R. T., Wu, S., \& Wrobel, J. S. (2010). Assessing postural control and postural control strategy in diabetes patients using innovative and wearable technology. J Diabetes $S c i$ Technol, 4(4), 780-791. 
Pozzo, T., Berthoz, A., \& Lefort, L. (1990). Head stabilization during various locomotor tasks in humans. I. Normal subjects. Exp Brain Res, 82(1), 97-106.

Prieto, T. E., Myklebust, J. B., Hoffmann, R. G., Lovett, E. G., \& Myklebust, B. M. (1996). Measures of postural steadiness: differences between healthy young and elderly adults. IEEE Trans Biomed Eng, 43(9), 956966. doi: $10.1109 / 10.532130$

Rocchi, L., Chiari, L., Cappello, A., \& Horak, F. B. (2006). Identification of distinct characteristics of postural sway in Parkinson's disease: a feature selection procedure based on principal component analysis. Neurosci Lett, 394(2), 140-145. doi:

10.1016/j.neulet.2005.10.020

Whitney, S. L., Roche, J. L., Marchetti, G. F., Lin, C. C., Steed, D. P., Furman, G. R., .. . Redfern, M. S. (2011). A comparison of accelerometry and center of pressure measures during computerized dynamic posturography: a measure of balance. Gait Posture, 33(4), 594-599. doi: 10.1016/j.gaitpost.2011.01.015 